\title{
Improving the Flight Endurance of a Separate-Lift-and-Thrust Hybrid through Gaussian Process Optimization
}

\author{
Francis Gregory $\mathrm{Ng}^{\mathrm{a}, *}$, Alvin Chua ${ }^{\mathrm{a}}$ \\ ${ }^{a}$ Mechanical Engineering Department, De La Salle University-Manila, 2401 Taft Avenue, Manila, 1004, Philippines \\ Corresponding author: * francis_gregory_ng@dlsu.edu.ph
}

\begin{abstract}
A separate-lift-and-thrust hybrid is a modified fixed-wing drone which includes quadcopter rotors. This results in the combined capability of forwarding flight as well as vertical take-off and landing (VTOL), making it a low-cost method that can deliver substantial gains in utility. Though this is a strong point compared to other types of VTOL drones, the hybrid design may incur a significant trade-off because added weight and drag can severely reduce the drone's flight endurance. This study attempts to mitigate the impact by improving the configuration of the selection and positioning parameters. Since drag estimations are costly, a Gaussian process optimization method was performed, as it is economical with respect to the required number of iterations. A set of arbitrarily selected components was prepared for use with the optimization method, recording the relevant performance data and constructing the CAD models of the components for use in simulations. The optimization method was able to increase the estimated flight endurance to 27.99 minutes, a significant improvement compared to a set of random configurations, which only yielded 9.54 minutes at best. The respectable result was obtained even though difficulties were experienced regarding the infeasible regions that arise from the many constraints. Future implementation of this optimization approach can be further improved. It may be worthwhile to utilize a low-fidelity model from the base fixed-wing drone simulations, in contrast with using an initial zero mean for the prior of the Gaussian process.
\end{abstract}

Keywords-Drone; Gaussian process; hybrid; kriging; optimization; simulation; unmanned aerial vehicle; VTOL.

\section{INTRODUCTION}

Unmanned aerial vehicles (UAV), also known as drones, are aircraft which do not require any pilot on board. This fact makes it quite versatile and opens the possibilities for many applications since it can economically carry small packages or lightweight instrumentation. Currently, two common drone types are the quadcopter and fixed-wing drones.

With four rotors that provide vertical thrust, the quadcopter may be tilted to move horizontally as well. The quadcopter's design makes it capable of vertical take-off and landing (VTOL), and it can also hover over a location. In most cases, though, the quadcopter drones are limited by energy concerns. On average, electrically-powered multi-copters only last for 10 to 30 minutes [1]. There has been research trying to utilize alternative energy sources such as gasoline engines [2] to increase flight endurance and allow for more useful applications. Alternatively, research has also been done to improve the quadcopter's performance efficiency by using variable-pitch propellers instead of traditional fixed-pitch propellers, but this increases the complexity of the control [3].
Meanwhile, maximizing the deployment of multiple drones to an environment would also be a useful management approach to this energy problem [4].

The energy-efficiency problem is less pronounced in the other type of UAV, the fixed-wing drone, which has relatively more payload capacity and flight range. Nonetheless, efforts have also been made regarding their power optimization [5]. Moreover, unlike the quadcopter, which is unstable in the event of a motor malfunction, the fixed-wing can still be safely landed like a glider. However, take-off and landing is generally more challenging as it requires a sufficiently long stretch of flat terrain, as well as reliable control. Another drawback of fixed-wing drones is the requirement to continuously move forward, meaning it cannot hover in place.

A clear approach to solve the deficiencies in the quadcopter and fixed-wing UAVs would be the deployment of a hybrid design which combines the good aspects of each, thus dramatically increasing the functionality of the drone. Multiple approaches exist like the tilt-rotor [6] where the rotors can rotate between vertical and forward thrust and the tilt-wing [7] where the wings rotate along with the rotors. The tail-sitter [8] may also be considered, though it only 
incorporates the forward thrust motors of the fixed-wing, meaning that it is capable of VTOL but lacks any hover capability. Other unique placements of vertical thrust rotors may also be possible such as in [9]. Nevertheless, the most simple and straightforward hybridization approach is the separate-lift-and-thrust (SLT) hybrid, as shown in Fig. 1, wherein the rotors are directly augmented to the fixed-wing to provide quadcopter functionality [10].

Despite the scarcity of research undertaken compared to the other drone hybrid types, the SLT hybrid is the most feasible hybridization attempt because it combines preexisting technology. SLT hybrid usage is certainly attainable as it can be implemented using currently available drone hardware and software. In fact, there is already an opensource control software called Ardupilot [11] that can be used to control this type of hybrid. The main concern now would be the development of the hardware itself, highlighting that greater weight and drag relative to the base fixed-wing drone may reduce the flight endurance.

This study aims to mitigate the flight endurance impact of hybridization through an improvement of the configuration. The parameters include the selection of components and the positioning of the rotor attachment on the drone. Ensuring competent flight times for the SLT hybrid will allow it to be the top choice in many drone applications.

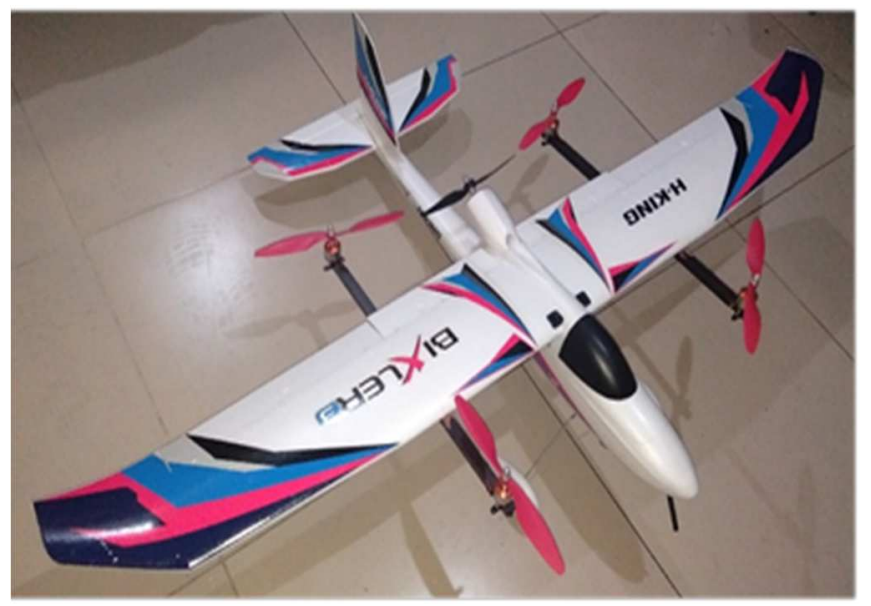

Fig. 1 Separate-lift-and-thrust hybrid drone

\section{MATERIAL AND MethoD}

The flight endurance is basically the endurance, $E$, of the battery. However, it is not merely proportional to the battery capacity, $C$, but also dependent on the empirical Peukert exponent, $n$. It is likewise important to consider the discharge time for which the capacity was tested, known as the battery hour rating, $R$. For the context of fixed-wing drones, the endurance calculation is shown in Eq. 1 [12]. It considers the efficiency of the propulsion system, $\eta_{\text {total }}$, and the power expended, equal to the product of the drag, $D$, and the flight speed, $U$. Since $R$ is typically equivalent to one hour for small rechargeable batteries [12] it is possible to simplify the equation. Nevertheless, the main challenge for evaluating flight endurance is the high cost for obtaining the drag value.

$$
E=R^{1-n}\left[\frac{\eta_{t o t a l} V C}{D U}\right]^{n}
$$

The methodology of the study is encapsulated in Fig 2. Since the drone will be hybridized, there are various alternative choices for the add-on components and many possible positions for attaching them. These parameters are to be manipulated to obtain configurations with enhanced flight endurance. To arrive at this, an optimization cycle is developed comprising of three phases: (i) the computer-aided design (CAD) where the 3D geometries are set up, (ii) the computational fluid dynamics (CFD) where the drag force is calculated, and (iii) the metamodel-based optimization (MBO) that identifies which configurations are worth exploring based on the expected improvement.

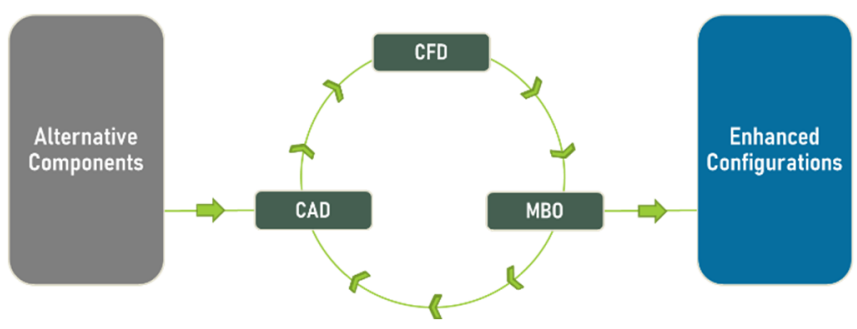

Fig. 2 Illustration of the optimization process.

\section{A. CAD Modeling}

The CAD is important because this enables the assimilation of real-life components into the optimization cycle, particularly in the CFD simulations which would follow. In the initialization, the various components are recreated. For this study, Volantex Ranger 2000 is the fixed-wing drone considered for hybridization, and it has been meticulously modeled using T-splines. It has a 2-meter wingspan with a recommended center of gravity (CG) at $70 \pm 5 \mathrm{~mm}$ from the wing's leading edge where it connects to the fuselage. This CG requirement is used as the basis for the midpoint of the quadcopter beam attachment.

Most drone components are housed within the fuselage, so these are not important to consider in the CAD setup. Rather, the focus is on the exterior components, which are the beam support, the motors, and the propellers. The former is simply modelled directly in the software while the latter two are more intricate. Thus, photogrammetry is utilized for improved results. Essentially, photogrammetry uses a set of images from various angles to reconstruct a $3 \mathrm{D}$ object. This is quite helpful for recreating the propellers given their unique twists which cannot simply be reverse engineered using parametric CAD. Further discussion on the implementation of photogrammetry for this purpose can be found in another research [13].

Moreover, to efficiently assemble the individual CAD models into whatever configuration is desired, an API script is constructed in Fusion 360 [14], the CAD software utilized for the study. The script essentially loads the selected components and references a dataset of joint locations to make proper connections. The script also handles other parameters used to configure the assembly, namely the beam length, the beam placement along the wing, and the drone's pitch angle. Afterwards, the assembly is exported after combining all the components into a single body. This prevents interference issues in the next phase, the CFD. 


\section{B. CFD Simulations}

Various parameters were applied in CAD to yield different configurations; with these, drag estimates will be obtained through CFD. However, the option of whether the propellers have an upward (pull) or downward (push) configuration has already been fixed. It has been argued by [15] that the latter option does not influence the airflow above the wing, so it is simply the superior option. This reduces the complexity of the problem and hence increases the focus in investigating the other parameters.

In conducting the flow simulation, Autodesk CFD [16] was the software of choice. This is from the same company as Fusion 360, and both are free for academic use. However, the main feature which makes the CFD software unique is the cloud processing capability where it outsources the simulation task to cloud servers. This typically comes with a hefty price, but fortunately, it is also free to use for academics. As such, this allows multiple simulations to be done in parallel without too much investment in computer hardware.

As recommended for use with the shear stress transport (SST) k-omega turbulence model, the simulations are set to have ten wall layers, a layer factor of 0.6, and a 1.25-layer gradation. This chosen turbulence model is more suitable for the task compared to the default general-purpose k-epsilon model. Essentially, it is better for flow separation predictions as it provides more accurate results at the boundary layer [17].

Another parameter in the CFD is the size adjustment which ranges from 0.2 to 5 , reflecting the size factor compared to the default mesh size. Unlike the wall layer settings where recommended values are available, this is more dependent on the specific simulation task. Therefore, a mesh independence test is performed on the base fixed-wing drone, where a value of 0.3 was found to provide a decent trade-off between accuracy and computational cost.

Targeting a particular flight speed for all simulations would be necessary so that improving the flight endurance will also equate to an improvement in the flight range. As the hybrid drone would be expected to perform similarly to the base fixed-wing drone, the latter serves as a standard for comparison to determine the target airspeed.

For a given aircraft, the most efficient state is when the angle of attack (AOA) maximizes the lift-to-drag ratio $L / D_{\max }$ (Fig. 3) [18]. Identically, this corresponds to where the total drag is minimized (Fig. 4) [18]. Constructing a plot like Fig. 4 from simulation data on the base fixed-wing drone, the relative wind is fixed at $0^{\circ}$ so that the angle of attack (AOA) is simply the drone's pitch angle. Then, a lift requirement must be determined. With a reasonable $25 \%$ payload fraction for the drone that has a battery weight of around $300 \mathrm{~g}$, the total weight becomes $20 \mathrm{~N}$.

Since it is not possible to identify beforehand the appropriate combinations of AOA and airspeed that could generate the needed lift, multiple simulations at each AOA must be done, and the drag then interpolated from the data. The resulting plot in Fig. 5 is the basis for setting the target speed of all simulations at $44 \mathrm{kph}$.

Moreover, a $L / D$ plot was likewise constructed (Fig. 6) and in accordance with theory, the maximum point of $5^{\circ}$ matched with the interpolation data behind Fig. 5. As these were separate sets of simulations, this agreement attests to the reliability of the result. As such, Fig. 6 was also used for estimating the required pitch angles of the hybrid configurations.

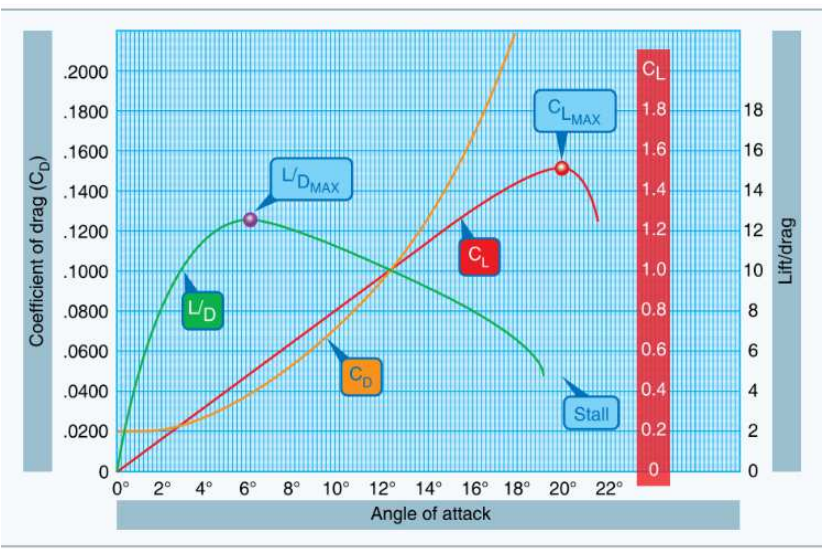

Fig. 3 Aircraft performance versus angle of attack [18].

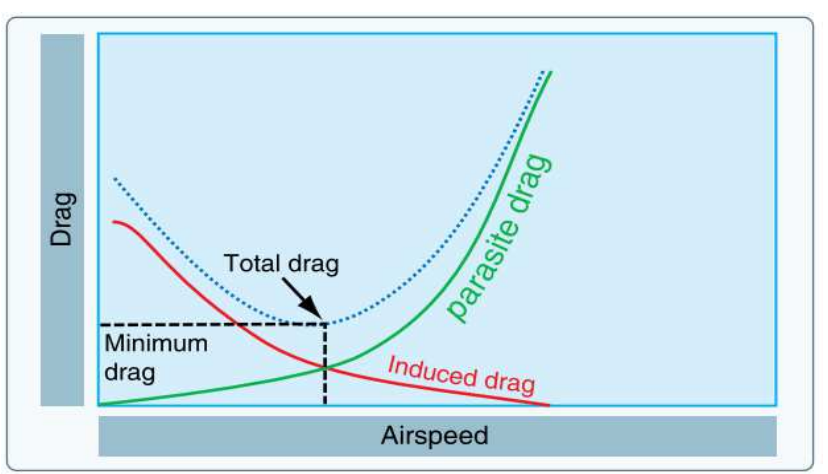

Fig. 4 Drag versus airspeed at constant lift [18].

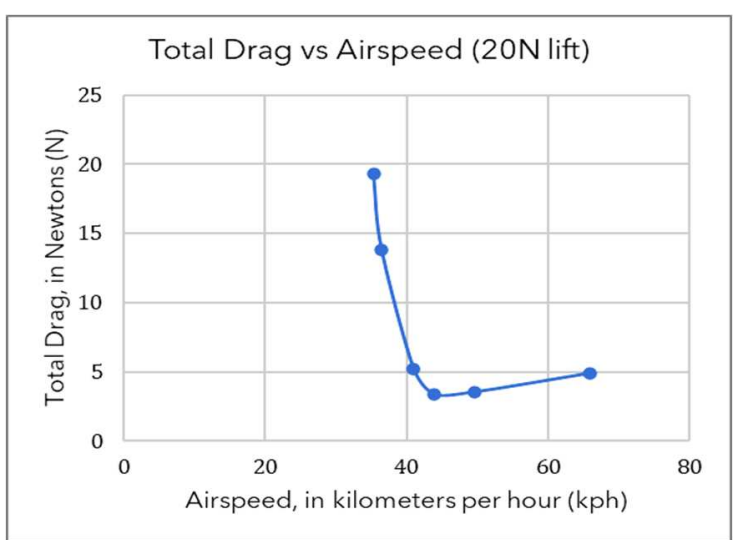

Fig. 5 Total drag versus airspeed of the fixed-wing drone at $20 \mathrm{~N}$ of lift.

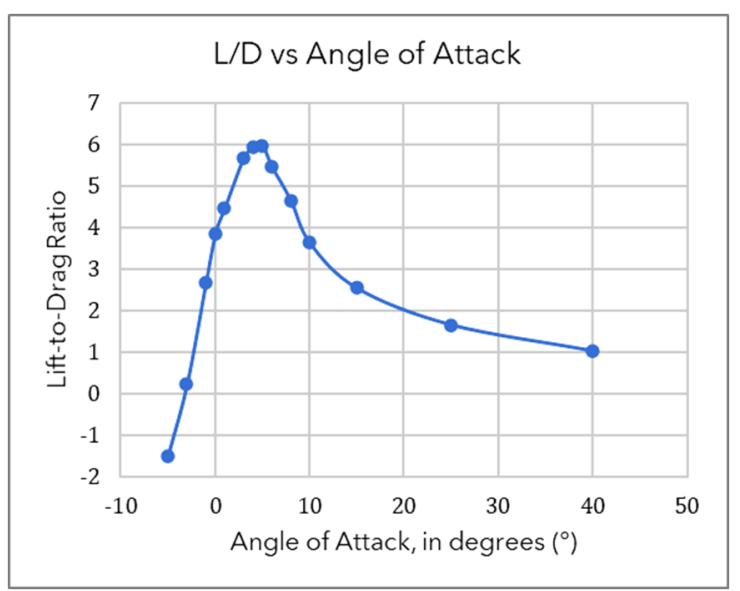

Fig. 6 Lift-to-drag ratio versus airspeed of the fixed-wing drone. 
A final consideration for the CFD is the possibility that during the forward flight of the hybrid drone, rotation may be induced on the quadcopter propellers. This has been investigated in [13], and it was concluded that no continuous rotation would result from the relative wind. This means that costly transient simulations are not necessary to determine the impact of the propellers on the drag as steady-state simulations would suffice. It has been identified that for a two-blade propeller, the steady-state position is at roughly $90^{\circ}$ to the relative wind, with a small angular deviation that may be attributed to the propeller design. For this study, however, the minor deviation is neglected, so the orientation is $90^{\circ}$ for all propellers.

\section{Gaussian Process Optimization}

The metamodel-based optimization method chosen for the study uses the GPyOpt package [19] in Python. The method utilizes a stationary Gaussian process with mean 0 , variance $\sigma^{2}$, and a covariance-based on the correlation function $R_{\theta}\left(\mathrm{x}_{i}, \mathrm{x}_{j}\right)$ where $\theta$ is a vector of covariance parameters [20]. The stationarity assumption merely asserts that the correlation is a function of the difference between inputs therefore $R_{\theta}\left(\mathrm{x}_{i}, \mathrm{x}_{j}\right)=R_{\theta}\left(\mathrm{x}_{i}-\mathrm{x}_{j}\right)$ [21]. The behavior of the Gaussian process is defined through the correlation function, also known as the kernel, for which the Matern $\left(\frac{5}{2}\right)$ kernel is typically used. With $h=\left|x_{1}-x_{2}\right|$, a 1-dimensional version of this kernel used in the study is shown in Eq. 2 [20].

$$
R(h)=\left(1+\frac{\sqrt{5} h}{\theta}+\frac{5 h^{2}}{3 \theta^{2}}\right) e^{-\frac{\sqrt{5} h}{\theta}}
$$

Fig. 7 illustrates a simple example of a 1-dimensional Gaussian process being used for optimization. The dashed curve indicates an unknown true model while the solid curve depicts the metamodel which is constructed from the observations. In the efficient global optimization (EGO) framework [22], an expected improvement (EI) acquisition function can be obtained, and this is maximized to determine the point of interest for future exploration.
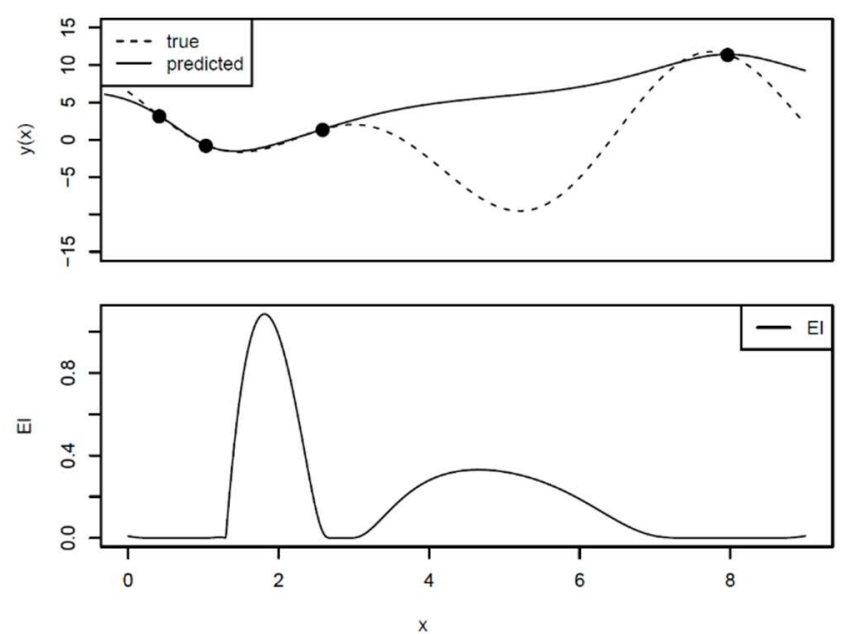

Fig. 7 Optimization using Gaussian process in the EGO framework [20].

The method is useful when the EI is significantly cheaper to evaluate compared to the actual function. This is quite appropriate for this study since CFD evaluations are very costly. Furthermore, batch iterations are done to leverage the parallel cloud simulation capability of the CFD software. Multiple points for evaluation are generated according to the local penalization method where the acquisition function undergoes a maximization-penalization loop (Fig. 8) [23]. This parallelization effectively reduces the timeframe and oversight required to conduct the simulations.
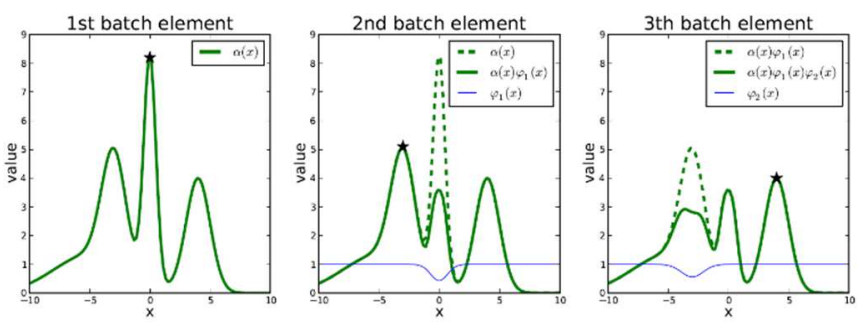

Fig. 8 Local penalization used to yield multiple suggested evaluations [23].

Even though the goal of improving component selection and positioning may seem simple, this requires handling a mix of categorical and continuous variables, unlike typical problems which only handle numerical values. For this purpose, it is necessary to apply the Gower kriging method by [20]. The existence of categorical variables also makes the constraints more difficult. So instead of a box-constrained problem with inequality constraints that apply to the entire domain, the problem requires inequalities that adapt to the categorical choices. For instance, the current draw and maximum recorded thrust will depend on the particular motor-propeller combination. Also, the minimum beam length and placement to avoid physical clashing are defined by the propeller diameter. In general, the constraints deal with physical clashing, thrust estimates, and battery compliance.

Since the CFD evaluations are the costliest step in the optimization cycle, constraints are pre-estimated to select which evaluations may return valid results. To obtain dependable calculations, performance data of all the motorpropeller combinations were obtained. But for constraints directly relating to the flow simulation results, it is necessary to rely on estimates, and these are sourced from the CFD results of the unhybridized drone. Thus, the constraints must be reevaluated afterwards to account for the hybrid configurations' simulation results. A penalty function is constructed to aggregate the percent violation for all constraints, assuming that each constraint is equally important.

It is also illustrated in Fig. 7 that Gaussian process optimization requires a starting set of samples to be able to suggest subsequent evaluations. Hammersley sequence sampling (HSS) was favored over Latin hypercube design (LHD) to generate the initial set of points. According to [24], HSS provides good uniformity for up to 40 dimensions while LHD is limited to one-dimensional uniformity due to random pairing for multiple dimensions. Moreover, HSS is not dependent on random permutations meaning that it generates the same configurations when parameters are maintained, reducing variability in the program, and enabling the easier isolation of mistakes during development.

\section{RESULTS AND DISCUSSION}

\section{A. Challenges in Implementation}

Applying constraints on the initial set of configurations significantly reduced the points for evaluation. For a sample size of 100 and 1000, there were 82 and 783 invalid configurations, respectively. Since the samples are distributed 
uniformly, this suggests that only roughly $20 \%$ of the entire domain is feasible for exploration.

The scarcity of feasible space renders it practically unattainable to avoid infeasible regions by means of penalty functions. An accurate metamodel would be useful, but this is not the aim of the optimization approach, but rather the exploration for expected improvement of objective values. Hence, the metamodel in use will not have a high enough resolution to identify the regions of feasibility.

Given this difficulty, the approach undertaken was to generate a larger HSS sampling and filter it through the constraint estimates. The exploration of the design space is then limited to the valid configurations identified through this procedure. This has the disadvantage of restricting the degree of freedom of the configurations, particularly in discretizing the continuous variables. It may be possible that a more robust approach can be used when faced with a domain that is dominated by infeasibility. Yet, the employed approach can still provide useful results, especially with large sampling sizes. Moreover, the best results may have feasible localities such that a non-discretized approach may be used to refine the optimization. However, this significantly increases the computational cost, so the possibility has not been explored and is left open for future investigation.

\section{B. Analysis of Performance}

An unhybridized fixed-wing drone is evaluated with varying battery selections, and the endurance values are plotted in the graph on Fig. 9. This serves as a point of comparison for the endurance enhancement of the hybrid drone. Given the trade-off of increased weight and drag for a VTOL-capable hybrid, the maximum endurance will be lower than the longest flight time estimate of 49.69 minutes, though it is not certain how closely it can meet this value.

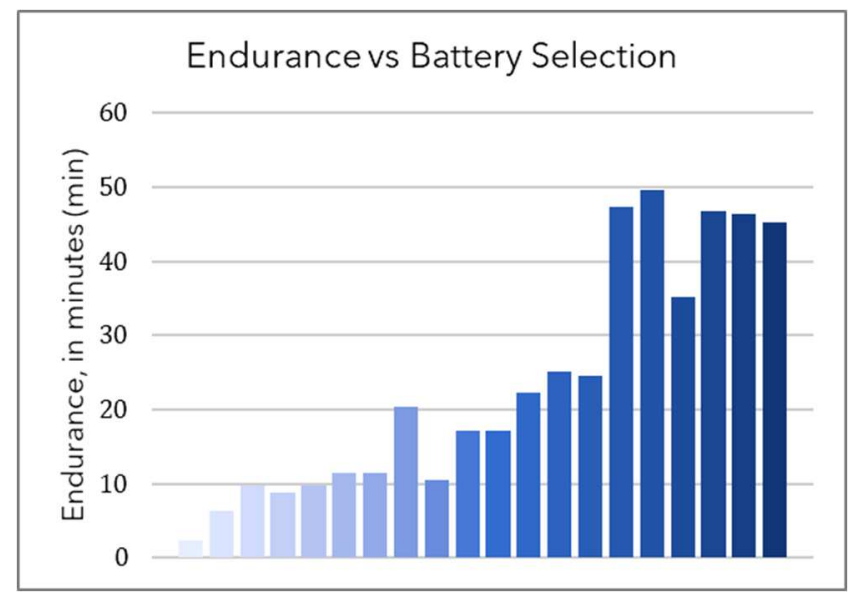

Fig. 9 Flight endurance of fixed-wing drone based on battery selection.

The optimization process resulted in a significant enhancement of endurance, as shown in Fig. 10. A respectable flight time of 27.99 minutes is estimated to be achieved given the best configuration found so far. Additional improvement might still be obtained based on the trend in the figure, but due to the limited computational effort allotted for the study, only three optimization batches were done. Nevertheless, the utility of this methodology has already been demonstrated as a batch of random configurations was found only to give 9.54 minutes at best. The method can certainly be further improved, but this is left as a topic of further study.

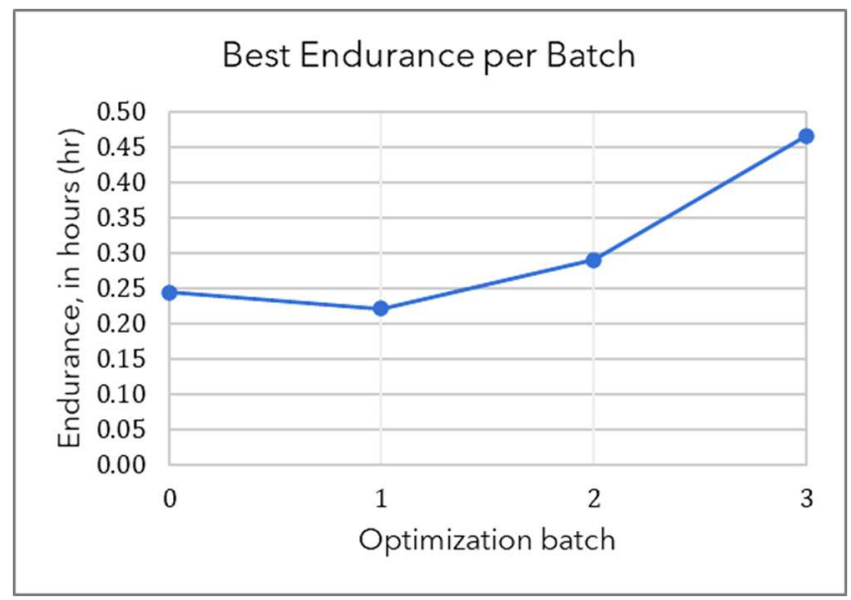

Fig. 10 Progression of batch optimization.

A significant issue with the execution of the Gaussian process optimization method is that the metamodel's prediction accuracy is quite low, having a surprisingly large value of 0.807 for the in-sample root-mean-square (RMS) error. This might be due to the high dimensionality of the problem, which has 6 input variables, four of which are categorical. Other than increasing the sample size, another strategy is needed to improve the prediction. Also, it could be beneficial to address the problem of the large extent of invalidity in the design space. Future study to handle both issues is recommended as this would surely boost the decent performance displayed by the current implementation.

\section{CONCLUSIONS}

Metamodel-based optimization, specifically Gaussian process optimization, has been employed to improve the separate-lift-and-thrust hybrid drone. With modifications made for using the Gower kriging method, the optimization can effectively operate on the component selection and positioning parameters, which are respectively categorical and continuous variables.

For obtaining sensible results, alternative components were arbitrarily selected, and their characteristic data were obtained. These were then modelled using CAD assisted by photogrammetry. Moreover, preliminary CFD analyses were done to set up the flight scenario of the SLT hybrid properly.

In the optimization, the design space was dominated by infeasible regions due to the various constraints. By restricting the search to pre-checked points generated from a space-filling design, the optimization was able to proceed. Cloud computing was leveraged to process multiple batches of simulations in parallel. Even though parallelization is less efficient than sequential sampling, a proper result was still obtained after a few iterations. The resulting configuration has almost $60 \%$ the endurance of the pure fixed-wing, which is significantly larger than the $20 \%$ obtained from an unplanned configuration. The metamodeling prediction quality is low, given the complexity of the design space but with insights from the results, a more effective $\mathrm{MBO}$ implementation can be done in the future. 
The optimization method performed well in this study, but to increase the utility of MBO, it would be better to yield a useful metamodel for predicting the performance of any configuration. A good approach to accomplish this would be to add a preliminary step to condition the design space. In contrast to an initial zero mean throughout, a far better prior for the Gaussian process can be constructed by using a lowfidelity model based on the lift and drag simulations of the base fixed-wing drone. A concluding step may also be considered where wind tunnel experiments further explore the promising optimization results, reducing the impact of numerical errors in the CFD simulations.

To increase the SLT hybrid drone's flight endurance, it can be better to also hybridize its power system. By utilizing combustion engines, high energy-density fuel can significantly improve the performance when the UAV is properly designed, with the electrical system still being used for redundancy, quick response, and possible solar power integration. Also, variable-pitch propellers may be used to increase the efficiency of the aircraft. By leveraging multiple ways to increase the endurance, a drone can remain in flight for exceptionally long periods of time.

\section{ACKNOWLEDGMENT}

The authors would like to thank the Philippines' Department of Science and Technology - Science Education Institute (DOST-SEI) and the Engineering Research and Development for Technology (ERDT). The valuable scholarship support aided in the completion of this study.

\section{REFERENCES}

[1] M. De Benedetti, F. D’Urso, G. Fortino, F. Messina, G. Pappalardo and C. Santoro, "A fault-tolerant self-organizing flocking approach for UAV aerial survey," Journal of Network and Computer Applications, vol. 96, pp. 14-30, Aug. 2017. doi: 10.1016/j.jnca.2017.08.004

[2] P. Tao, "Design, Prototyping and Autonomous Control of GasolineEngine Variable-Pitch Quadcopter," M. Eng. thesis, National University of Singapore, Singapore, Singapore, May 2016.

[3] I. R. Mcandrew, E. Navarro and K. Witcher (2018), "Propeller Design Requirements for Quadcopters Utilizing Variable Pitch Propellers," International Journal of Materials, Mechanics and Manufacturing, vol. 6(1), pp. 51-56, Feb. 2018. doi:10.18178/ijmmm.2018.6.1.346

[4] D. Zorbas, L. D. P. Pugliese, T. Razafindralamb and F. Guerriero, "Optimal drone placement and cost-efficient target coverage," Journal of Network and Computer Applications, vol. 75, pp. 16-31. Aug. 2016. doi: $10.1016 /$ j.jnca.2016.08.009

[5] P. G. Sai, C. S. Rani and U. R. Nelakuditi, "Implementation of Power Optimization Technique for UAVs," in PMME 2016 Implementation, 2018, paper 5, pp. 132-137. doi: 10.1016/j.matpr.2017.11.063

[6] W. Lu, D. Zhang, J. Zhang, T. Li and T. Hu, "Design and Implementation of a Gasoline-electric Hybrid Propulsion System for a Micro Triple Tilt-rotor VTOL UAV," in 2017 IEEE 6th Data Driven Control and Learning Systems Conference, 2017, pp. 433-438. doi:10.1109/DDCLS.2017.8068112
[7] E. Cetinsoy, S. Dikyar, C. Hancer, K. T. Oner, E. Sirimoglu, M. Unel and M. F. Aksit, "Design and construction of a novel quad tilt-wing UAV," Mechatronics, vol. 22(6), pp. 723-745, May 2012. doi: 10.1016/j.mechatronics.2012.03.003

[8] M. Hochstenbach, C. Notteboom, B. Theys and J. De Schutter, "Design and Control of an Unmanned Aerial Vehicle for Autonomous Parcel Delivery with Transition from Vertical Take-off to Forward Flight - VertiKUL , a Quadcopter Tailsitter," International Journal of Micro Air Vehicles, vol. 7(4), pp. 395-406, Dec. 2015. doi:10.1260/1756-8293.7.4.395

[9] S. Carlson, "A Hybrid Tricopter/Flying-Wing VTOL UAV," in 52nd Aerospace Sciences Meeting, 2014, paper 2014.0016, pp. 1-11. doi:10.2514/6.2014-0016

[10] P. T. Dewi, G. S. Hadi, M. R. Kusnaedi, A. Budiyarto and A. Budiyono, "Design of Separate Lift and Thrust Hybrid Unmanned Aerial Vehicle," The Journal of Instrumentation, Automation and Systems, vol. 2(2), pp. 45-51, Oct. 2015. doi:10.13140/RG.2.1.4900.9681

[11] ArduPilot. (2016) QuadPlane Overview. [Online]. Available: $\mathrm{http}: / /$ ardupilot.org/plane/docs/quadplane-overview.html

[12] L. W. Traub, "Range and Endurance Estimates for Battery-Powered Aircraft," Journal of Aircraft, vol. 48(2), pp. 703-707, Apr. 2011. doi:10.2514/1.C031027

[13] F.G. Ng and A. Chua, "Simulating the Effect of Non-Axial Airflow on the Motion of an Undriven Propeller," International Journal of Advanced Trends in Computer Science and Engineering, vol. 9(1), pp. 153-159, Feb. 2020. doi:10.30534/ijatcse/2020/23912020

[14] Autodesk Inc. (2020) Fusion 360 free 3D CAD/CAM design software for students, educators, and academic institutions. [Online]. Available: https://www.autodesk.com/products/fusion-360/students-teacherseducators

[15] R. Czyba, M. Lemanowicz, Z. Gorol and T. Kudala, "Construction Prototyping, Flight Dynamics Modeling, and Aerodynamic Analysis of Hybrid VTOL Unmanned Aircraft," Journal of Advanced Transportation, vol. 2018(7040531), pp.1-15, Oct. 2018. doi: $10.1155 / 2018 / 7040531$

[16] Autodesk Inc. (2020) CFD Ultimate. [Online]. Available: https://www.autodesk.com/education/free-software/cfd-2

[17] F. Menter, "Review of the shear-stress transport turbulence model experience from an industrial perspective," International Journal of Computational Fluid Dynamics, vol. 23(4), pp. 305-316, Jan. 2009. doi: $10.1080 / 10618560902773387$

[18] Federal Aviation Administration, Pilot's handbook of Aeronautical Knowledge: Aerodynamics of Flight. Federal Aviation Administration, 2016.

[19] The GPyOpt authors. (2016) GPyOpt: A Bayesian Optimization framework in Python. [Online]. Available: https://github.com/SheffieldML/GPyOpt

[20] M. Halstrup, "Black-box optimization of mixed discrete-continuous optimization problems" Dr. rer. nat. thesis, TU Dortmund University, Dortmund, Germany, Jul. 2016.

[21] M. Osborne, "Bayesian Gaussian Processes for Sequential Prediction, Optimisation and Quadrature," Ph. D. thesis, University of Oxford, Oxford, England, Mar. 2010. doi:10.1128/JVI.78.14.7590-7601.2004

[22] D. R. Jones, M. Schonlau and W. J. Welch, "Efficient Global Optimization of Expensive Black-Box Functions," Journal of Global Optimization, vol. 13, pp. 455-492, Jun. 1998. doi:10.1023/A:1008306431147

[23] J. González, Z. Dai, P. Hennig and N. Lawrence, "Batch bayesian optimization via local penalization" in Proceedings of the 19th International Conference on Artificial Intelligence and Statistics, 2016, pp. 648-657.

[24] N. S. Dige, "Efficient Sampling Algorithm for Optimization under Uncertainty," M. Sc. thesis, University of Illinois. Chicago, Illinois, Jun. 2016. 